\title{
Control of Inward Rectifier K Channel Activity by Lipid Tethering of Cytoplasmic Domains
}

\author{
Decha Enkvetchakul, lana Jeliazkova, Jaya Bhattacharyya, and Colin G. Nichols \\ Department of Cell Biology and Physiology, Washington University School of Medicine, St. Louis, MO 63110
}

\begin{abstract}
Interactions between nontransmembrane domains and the lipid membrane are proposed to modulate activity of many ion channels. In Kir channels, the so-called "slide-helix" is proposed to interact with the lipid headgroups and control channel gating. We examined this possibility directly in a cell-free system consisting of KirBac1.1 reconstituted into pure lipid vesicles. Cysteine substitution of positively charged slide-helix residues (R49C and $\mathrm{K} 57 \mathrm{C}$ ) leads to loss of channel activity that is rescued by in situ restoration of charge following modification by $\mathrm{MTSET}^{+}$or $\mathrm{MTSEA}^{+}$, but not MTSES ${ }^{-}$or neutral MMTS. Strikingly, activity is also rescued by modification with long-chain alkyl-MTS reagents. Such reagents are expected to partition into, and hence tether the side chain to, the membrane. Systematic scanning reveals additional slide-helix residues that are activated or inhibited following alkyl-MTS modification. A pattern emerges whereby lipid tethering of the $\mathrm{N}$ terminus, or $\mathrm{C}$ terminus, of the slidehelix, respectively inhibits, or activates, channel activity. This study establishes a critical role of the slide-helix in Kir channel gating, and directly demonstrates that physical interaction of soluble domains with the membrane can control ion channel activity.
\end{abstract}

\section{INTRODUCTION}

For many ion channels, lipid membrane composition is proposed to be an important regulator of channel gating (Hilgemann and Ball, 1996; Shyng and Nichols, 1998; Baukrowitz et al., 1998; Runnels et al., 2002; Rohacs et al., 2005; Suh and Hille, 2005) but physical evidence for direct interactions of channel domains with lipids is lacking. In the inwardly rectifying $\mathrm{K}$ (Kir) channels, a large and widespread channel family that modulates excitability throughout the organism, channel gating is proposed to be controlled by the "slide-helix," a novel feature seen in the crystal structure of the bacterial Kir homologue KirBac 1.1 (Kuo et al., 2003). The slide-helix is an $\alpha$-helical segment immediately preceding the first transmembrane segment, and is predicted to lie parallel to the membrane, in the vicinity of the phospholipid headgroups (Fig. $1 \mathrm{~A}$ ). It has been proposed that the slide-helix interacts with the phospholipid headgroups and forms a link between the binding site for gating molecules (such as ATP in Kir6.2) and the ligand-dependent channel gate (Kuo et al., 2003), which is likely to be at the crossing point of the second transmembrane helix bundle. Mutations of Kir channels within the region of the slide-helix are known causes of inherited Kir channel disorders (Schulte et al., 1999; Plaster et al., 2001; Schulze et al., 2003; Gloyn et al., 2004).

In this study, we examine the role of interactions between the lipid membrane and the slide-helix in control of Kir channel gating in a pure channel-lipid system.

Correspondence to D. Enkvetchakul: denkvetc@wustl.edu; or C.G. Nichols: cnichols@wustl.edu
The results provide direct demonstration of control of channel activity by physical interaction of a nontransmembrane domain of the channel with the lipid membrane.

\section{MATERIALS AND METHODS}

Methods are essentially as described previously (Enkvetchakul et al., 2004). KirBac1.1 was cloned from genomic DNA of Burkholderia pseudomallei by PCR and subcloned into the pQE60 vector (QIAGEN) as a C-terminal six histidine-tagged construct. Single cysteine mutations were made using the Quikchange Sitedirected Mutagenesis Kit (Stratagene). All mutants were verified by DNA sequencing.

For protein purification, KirBac1.1 in pQE60 was expressed in BL21* (DE3) cells induced with isopropyl $\beta$-D-thiogalactopyranoside. Bacteria were lysed by a freeze-thaw cycle, incubated for $2-4 \mathrm{~h}$ in resuspension buffer $(50 \mathrm{mM}$ Tris- $\mathrm{HCl}, \mathrm{pH} 8.0,150 \mathrm{mM} \mathrm{KCl}$, $250 \mathrm{mM}$ sucrose, $10 \mathrm{mM} \mathrm{MgSO} 4$ ) with $30 \mathrm{mM}$ decylmaltoside (Anatrace), and then centrifuged at $30,000 \mathrm{~g}$ for $30 \mathrm{~min}$. The supernatant was mixed with $\sim 0.2-0.4 \mathrm{ml}$ cobalt beads, washed with 40 bed volumes of wash buffer $(50 \mathrm{mM}$ Tris-HCl, $\mathrm{pH} 7.4,150 \mathrm{mM}$ $\mathrm{KCl}, 10 \mathrm{mM}$ imidazole, and $5 \mathrm{mM}$ decylmaltoside), and KirBac1.1 was eluted with $2 \mathrm{ml}$ of wash buffer containing $500 \mathrm{mM}$ imidazole. Proteins were concentrated using $30-\mathrm{kD}$ centrifugal filters (Millipore) to $0.5-5 \mathrm{mg} / \mathrm{ml}$.

For $\mathrm{Rb}^{+}$flux assay, disposable polystyrene columns (Pierce Chemical Co.) were packed with Sephadex G-50 (fine) beads (1 ml), swollen overnight in buffer A or B (buffer A: $450 \mathrm{mM} \mathrm{KCl}, 10 \mathrm{mM}$ HEPES, $4 \mathrm{mM}$ NMG, pH 7; buffer B: $450 \mathrm{mM}$ sorbitol, $10 \mathrm{mM}$ HEPES, $4 \mathrm{mM}$ NMG, $50 \mu \mathrm{M} \mathrm{KCl}, \mathrm{pH} 7.0$ ). 2-3 $\mu \mathrm{g}$ of purified protein per $\mathrm{mg}$ of total lipid was added to a CHAPS $(37 \mathrm{mM})$ solubilized mixture of phosphatidylethanolamine:phosphatidylglycerol (3:1, Avanti Polar Lipids, Inc., $10 \mathrm{mg}$ total lipid per ml) in buffer A and incubated $30 \mathrm{~min}$. Column A (with Sephadex beads in buffer A) were partially dehydrated by spinning at 3,000 rpm in a Beckman TJ6 centrifuge. Liposomes were formed by spinning $100 \mu \mathrm{l}$ 
A

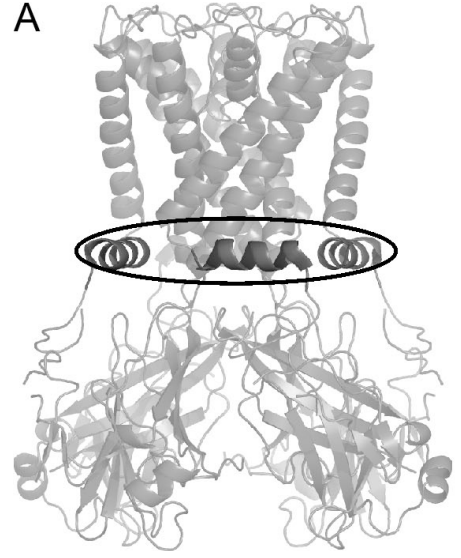

B

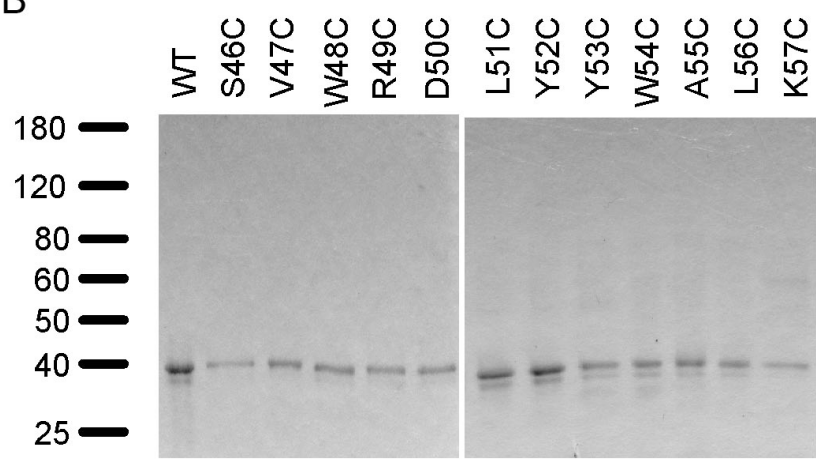

Figure 1. (A) Ribbon model of KirBac1.1 crystal structure, demonstrating the location of the slide-helices, emphasized in dark gray. (B) SDS-PAGE of purified WT KirBacl.1 protein and cysteine-substituted mutants, stained with Coomassie blue. of detergent-solubilized lipid/protein mixture through the partially dehydrated column A at 2,500 rpm. Extraliposomal solution was exchanged for buffer B by centrifugation through a partially dehydrated column $\mathrm{B}$. The assay was initiated by adding $400 \mu \mathrm{l}$ of buffer B with $1-5 \mu \mathrm{M}^{86} \mathrm{Rb}^{+} .50-60-\mu$ l aliquots of the radioactive mixture were taken at time points indicated, and extraliposomal ${ }^{86} \mathrm{Rb}^{+}$removed by passage over a $0.5-\mathrm{ml}$ Dowex cation exchange column in the $\mathrm{NMGH}^{+}$form. Samples were mixed with scintillation fluid and counted in a liquid scintillation counter. Valinomycin was used to assay maximal ${ }^{86} \mathrm{Rb}^{+}$uptake. MTS modification of KirBac1.1 cysteine mutants was prepared by incubating purified mutant protein in $100 \mu \mathrm{M}$ MTS reagent for $\sim 30$ min before reconstitution.

In all figures, values indicated are mean \pm SEM, except Fig. 3, which shows values $\pm 95 \%$ confidence intervals.

\section{RESULTS AND DISCUSSION}

\section{Charged Residues in the Slide-Helix Control Channel Activity}

To probe the role of the slide-helix in controlling channel activity, we systematically mutated each slide-helix residue in KirBac1.1 to cysteines, and examined the consequences of these mutations and subsequent in situ modification on channel activity. Of initial interest were the positively charged residues $\mathrm{R} 49$ and $\mathrm{K} 57$, since positive charges in this vicinity might regulate channel activity by interaction with negatively charged headgroups on the membrane lipids, or with negative charges in the cytoplasmic domain. Purified wild-type KirBac1.1 (which is cysteineless) (Fig. $1 \mathrm{~B}$ ) shows robust channel activity when reconstituted into 3:1 POPE:POPG membranes (Enkvetchakul et al., 2004) (Fig. 2), but when R49 and K57 were mutated to cysteine, reconstituted KirBac1.1[R49C] and KirBac1.1[K57C] channel activity was negligible (Fig. 2). However, activity was restored following covalent modification by the positively charged MTSEA $^{+}$, or MTSET ${ }^{+}$(Fig. 2), indicating that a positive charged side chain is necessary and sufficient for channel activity. By contrast, channel activity was not restored by negatively charged MTSES or neutral methyl-MTS (Fig. 2). These results are entirely consistent with the side chains of R49 and K57 interacting electrostatically with negative charges located either within the channel or with negatively charged lipid headgroups to maintain channel activity.

\section{Lipid Tethering Can Rescue Charge Neutralization}

Control of gating by channel-membrane interactions has been postulated for multiple eukaryotic channels (Enkvetchakul and Nichols, 2003; Suh and Hille, 2005). Implicit in this is the simple, but untested, concept that electrostatic "anchoring" of cytoplasmic, nontransmembrane domains to the membrane provides the energetic push or pull to stabilize open or closed states. We hypothesized that if a physical interaction of the slidehelix with the lipid membrane is needed for channel activity, then tethering of the slide-helix through hydrophobic anchors that favorably partition into the lipid membrane should have the same effect. The cysteine mutation allows us to take advantage of MTS reagents to attach just such a hydrophobic anchor. Modification by alkyl MTS reagents attaches a long hydrophobic side chain to the cysteine, which in turn will be expected to partition into, and hence "tether" the side chain to, the lipid membrane. KirBac[R49C] protein was modified by decyl-MTS before reconstitution into liposomes. A striking rescue of $\mathrm{R} 49 \mathrm{C}$ activity was indeed achieved by modification with decyl-MTS (Fig. 2 B).

We then systematically mutated each residue of the slide-helix to cysteine to assess the effect on channel activity of attaching a lipid tether at different points along the length of the slide-helix. Cysteine substitution was tolerated at many residues, without loss of channel activity, but activity was abolished for nearly all residues in the N-terminal end of the helix (residues 52-55 and 57, Fig. $3 \mathrm{~A}$ ). We then examined channel activity after modification of these residues with decyl-MTS before reconstitution into liposomes. Decyl-MTS modification caused significant channel activation at the predicted exposed residues V47C, R49C, and L51C in the N-terminal segment of the slide-helix (Fig. 3). Strong inhibition by decyl-MTS modification was seen for L56C, which, in contrast to the activated residues, is normally buried 

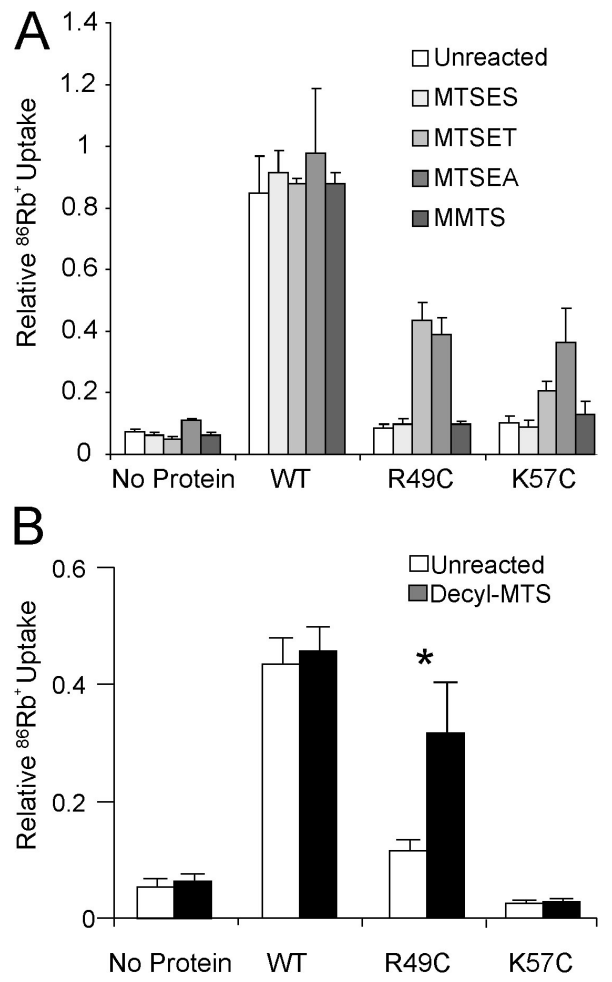

Figure 2. (A) Rescue of mutant KirBac1.1 channels R49C or K57C, by restoration of positive charges after modification with MTSEA $^{+}$ or $\mathrm{MTSET}^{+}$, but not with MTSES ${ }^{-}$or MMTS. (B) Rescue of R49C channel activity after modification with decyl-MTS. In both panels, ${ }^{86} \mathrm{Rb}^{+}$uptake was measured at $2 \mathrm{~min}$ in liposomes reconstituted with purified KirBac1.1 protein. Proteins were incubated with $100 \mu \mathrm{M}$ MTS reagents for 30-60 min before incorporation into liposomes. Rubidium uptake was normalized to maximal uptake as measured by valinomycin ( $n=3-6$; * $\mathrm{P}>0.05$, versus unmodified).

within the protein interior at the C-terminal end of the slide-helix (Fig. 3).

\section{Channel Activity Is Proportional to Degree of Tethering}

If tethering of residues on the slide-helix to the lipid membrane is indeed responsible for modulation of channel activity, this effect is expected to vary with the hydrophobicity of the attached tether. Tethers with increased hydrophobicity are expected to partition more strongly into the lipid membrane, in proportion to the length of the attached alkyl chain. In accordance with the prediction, alkyl-MTS modification of R49C (Fig. 4 A) or V47C (Fig. 4 B) demonstrated an essentially monotonic increase in channel activity with increasing chain length. In contrast, no effect was seen with alkylMTS modification of K57C (Fig. 4 A), consistent with the notion that lipid tethering of residue 57 does not promote channel opening. L51C (Fig. $4 \mathrm{C}$ ) and L56C (Fig. $4 \mathrm{D}$ ) demonstrated a more complex behavior. L51C exhibited progressively greater activation with alkyl chains longer than butyl, consistent with channel activity depending on the degree of lipid tethering of
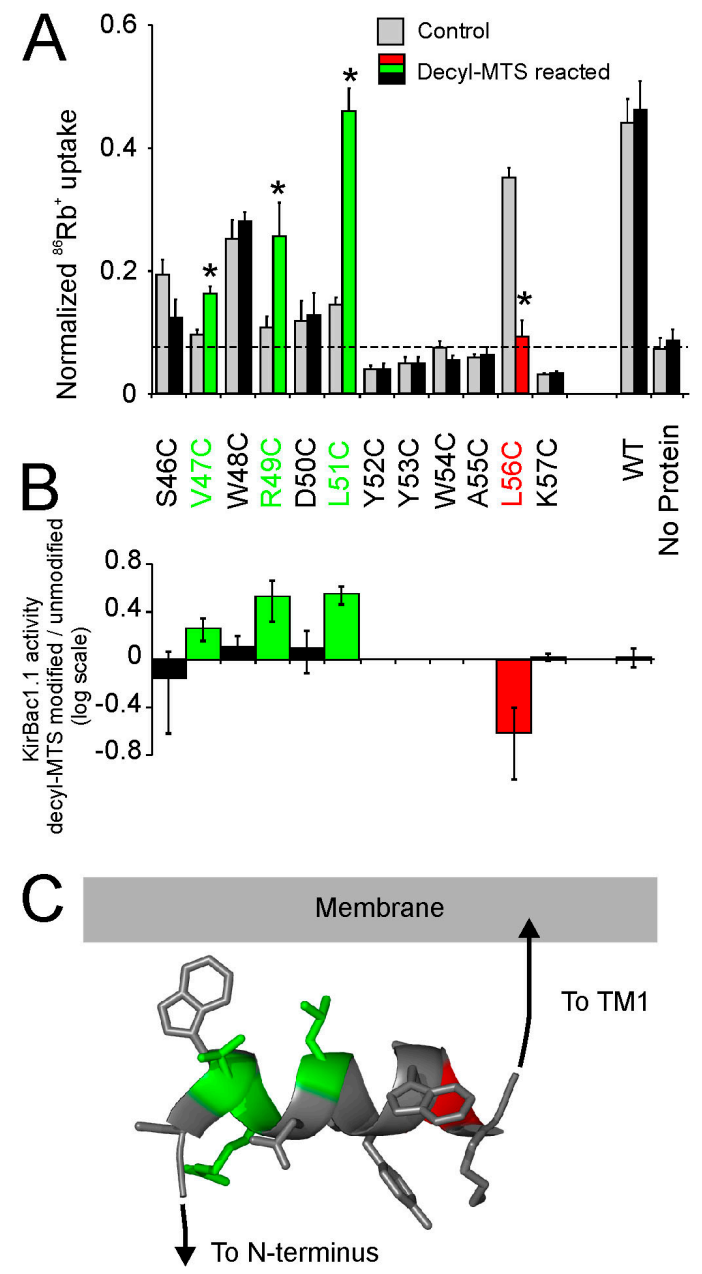

Figure 3. KirBac1.1 channel activity modulated by covalent attachment of hydrophobic decyl anchors to the slide-helix. (A) Rubidium uptake of KirBac1.1 cysteine mutants (gray bars), or mutants reacted with decyl-MTS (red/green/black bars). KirBac1.1 protein was incubated with $100 \mu \mathrm{M}$ decyl-MTS for $30 \mathrm{~min}$ before incorporation into liposomes. Significantly altered activity is indicated by asterisks ( $n=3-6$; *, $\mathrm{P}>0.05$, versus unmodified). (B) Log ratio of channel activity before and after modification with decyl-MTS. Mutants that have no channel activity before and after modification (as compared with liposomes) are excluded. Error bars represent 95\% confidence interval. (C) Ribbon model of the KirBac1.1 slide-helix, with side chains shown in stick format. Residues are colored either green or red to represent increased or decreased channel activity after decyl-MTS modification, or as gray for mutants that had unchanged or no activity.

this residue to the lipid membrane (Fig. 4 C). However, weak activation of channel activity is also seen in both L51C and L56C with shorter (methyl and propyl) chains. This might be expected, however, considering that the native residue is leucine, and addition of a short alkyl chain will effectively restore the hydrophobic bulk normally provided by the leucine side chain. Longer chain alkyl-MTS modification resulted in monotonic inhibition of L56C, indicating that tethering of this residue at the C-terminal end of the slide-helix causes closure of the channel. In each case, for V47C, R49C, 


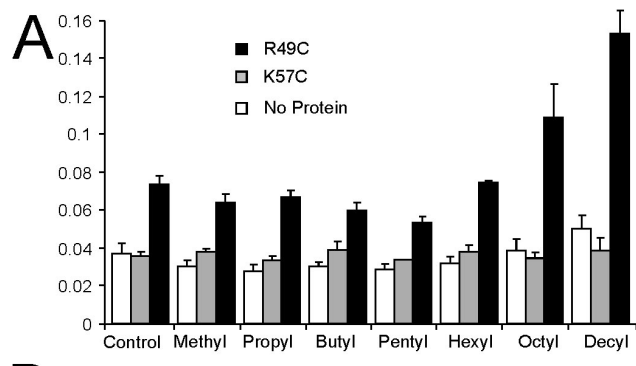

B

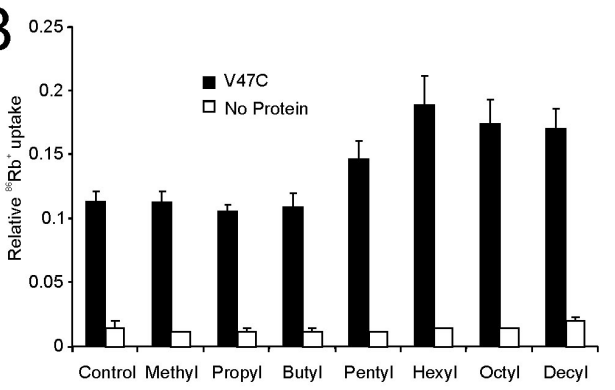

C

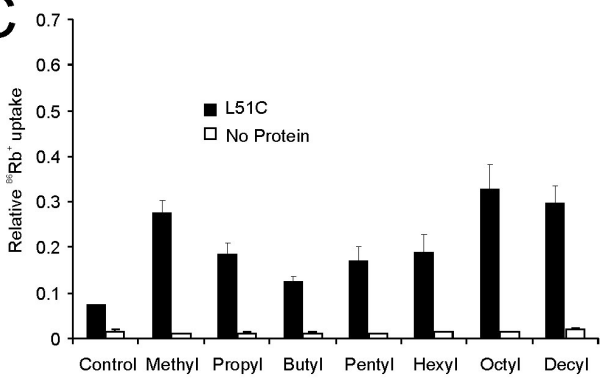

$\mathrm{D}$

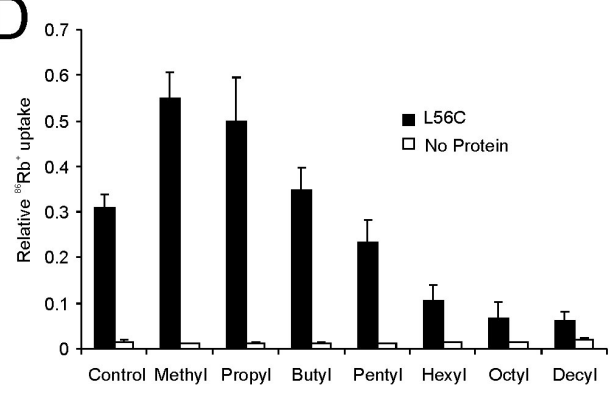

Figure 4. KirBac1.1 channel activity dependence of alkyl side chain length. Purified KirBac1.1 channel activity was measured in a rubidium uptake assay for (A) R49C, K57C, (B) V47C, (C) L51C, or (D) L56C. Proteins were incubated with $100 \mu \mathrm{M}$ alkyl-MTS reagent for $30 \mathrm{~min}$ before incorporation into liposomes.

L51C, and L56C, progressive activation or inhibition was seen, dependent on the length of the attached alkyl chain and on the increased octanol-water partition coefficient of longer alkyl groups (Khadikar et al., 2003). This argues strongly that the effect on channel activity is directly proportional to the ability of the alkyl side chain to partition into the lipid, and thereby to tether the residue to the membrane.

$\mathrm{PIP}_{2}$ is a potent inhibitor of KirBac1.1 channel activity (Enkvetchakul et al., 2005), and as hypothesized for eukaryotic Kir channels, PIP $_{2}$ may interact through its negatively charged headgroup with residues in the

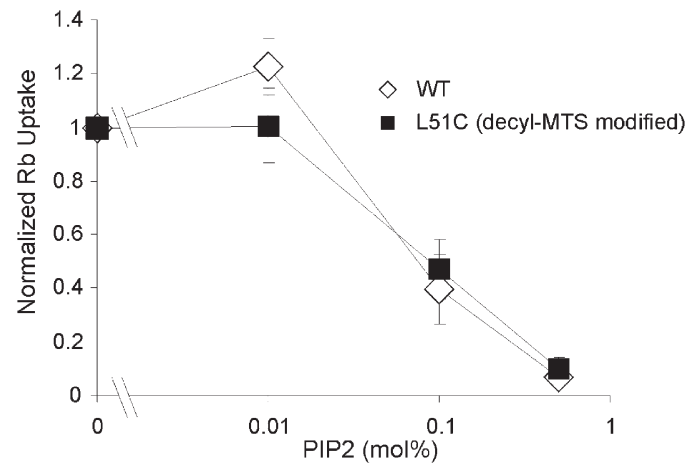

Figure 5. $\mathrm{PIP}_{2}$ inhibition of KirBac1.1 WT or L51C channel activity. Purified KirBac protein was reconstituted into liposomes formed with varying percentage of $\mathrm{PIP}_{2}$. Uptake was measured at $30 \mathrm{~s}$. L51C protein was incubated with $100 \mu \mathrm{M}$ decyl-MTS for $30 \mathrm{~min}$ before incorporation into liposomes.

$\mathrm{N}$ and $\mathrm{C}$ termini. We investigated the effect of lipid tethering of the slide-helix to the membrane on the potency of $\mathrm{PIP}_{2}$ inhibition of KirBac1.1 channel activity, and focused on decyl-MTS-modified L51C, which demonstrated the greatest degree of activation (Fig. 3 A). L51C was incubated with decyl-MTS before reconstitution, and the activity of L51C and WT KirBac1.1 channels were measured in liposomes formed with varying concentrations of $\mathrm{PIP}_{2}$. The effect of lipid tethering of L51C did not alter PIP 2 sensitivity, and both WT and L51C were half-maximally inhibited by membrane $\mathrm{PIP}_{2}$ at $\sim 0.1 \mathrm{~mol} \%$ (Fig. 5).

\section{Role of the Slide-Helix in Kir Channel Gating}

The KirBac1.1 crystal (Kuo et al., 2003) provides a structural model for understanding the molecular basis of gating in Kir channels as a whole. In this structure, KirBac1.1 channels are presumed to be in the closed state, with the central ion permeation pathway occluded by the side chain of the phenylalanine at position 146 . Gating has been proposed to occur by coupling of the movement of the cytoplasmic domains to the transmembrane domains through the amphipathic slidehelices, which are predicted to lie along the plane of the lipid membrane. Consistent with this idea, mutation of residues in the slide-helix region of eukaryotic Kir channels can have a profound effect on channel gating, and can underlie human disease (Schulte et al., 1999; Plaster et al., 2001; Schulze et al., 2003; Gloyn et al., 2004). Potentially this results from altering slide-helix interactions with phospholipid headgroups of the lipid membrane (Plaster et al., 2001; Schulze et al., 2003). However, the complex system of the eukaryotic cell membrane precludes any rigorous assessment of channellipid interactions, and these ideas have remained essentially unaddressable. The above experiments show clearly that manipulations of the slide-helix can control KirBac1.1 channel activity, and that tethering of 

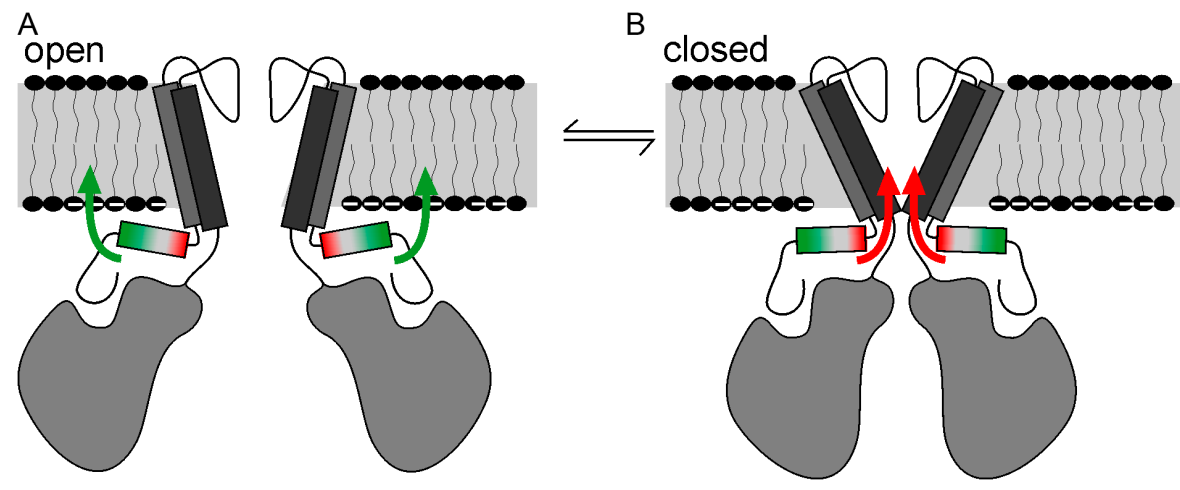

Figure 6. Cartoon model of the role of the slide-helix in Kir channel gating. Movements of the slide-helix with respect to the membrane are associated with increased stability of the open or closed state of the channel. (A) Tethering of the C-terminal end to the membrane, which may involve rotation, displacement, or tilting (green arrows), facilitates opening of the channel. In contrast, (B) tethering of the C-terminal end (red arrows) favors the closed state of the channel. slide-helix residues to the membrane can control channel activity directly. Furthermore, the data suggest that specific movements of the slide-helix are involved. KirBac1.1 channels are activated by lipid tethering of residues in the $\mathrm{N}$ terminus of the slide-helix to the membrane by addition of alkyl side chains, and are inhibited by lipid tethering of a normally buried residue at the $\mathrm{C}$ terminus of the slide-helix. Based on this, we propose that slide-helix movements are involved in Kir channel gating (Fig. 6), whereby movement of the N- or C-terminal ends with respect to the lipid membrane results in increased stability of the open or closed state of the channel, respectively. The slide-helix is intimately linked with the cytoplasmic C-terminal domain through a $\beta$-sheet association of the $\mathrm{N}$ terminus (Kuo et al., 2003). This makes it intuitively clear how movement of the cytoplasmic domain, through binding of ligands, could result in movement of the slide-helix, and we propose that binding of ligands to Kir channel cytoplasmic domains will be coupled to gating movements in the transmembrane domains through similar movement of the slide-helix.

We have shown that the positively charged $\mathrm{R} 49$ residue is necessary for channel activity, and that restoration of charge in the R49C mutant by MTS modification rescues activity. We suggest that $\mathrm{R} 49$ may interact with membrane phospholipids, consistent with the finding that lipid tethering of this residue and of nearby residues can activate the channel. Interestingly, primary sequence alignment of the KirBacs reveals that the position homologous to R49 in KirBac1.1 tends to be conserved as a basic residue. However, KirBac3.1 has a neutral leucine or positively charged aspartate (depending on the alignment) at the homologous position, and is an inactive/low activity channel (Sun et al., 2006), consistent with the findings that loss of a positive charge at residue 49 results in decreased KirBac1.1 channel activity.

The residue homologous to position K57 of KirBac1.1, however, is poorly conserved within the KirBac family, and a positively charged residue at this position is not necessary for activity for other members of the family, at least as assayed by growth rescue in $\mathrm{K}$ auxotrophic bacteria (Sun et al., 2006). We speculate that this positively charged residue may be important for structural integrity of the channel through its interaction with other charged residues within the KirBac1.1 protein in addition to the lipid membrane.

The findings of this paper are not inconsistent with our previously proposed mechanisms of $\mathrm{PIP}_{2}$ inhibition (Enkvetchakul et al., 2005). PIP $_{2}$ likely binds to several residues in the cytoplasmic domain of eukaryotic Kir channels, including residues located outside of the slide-helix in the $\mathrm{N}$ and $\mathrm{C}$ termini. This binding has been hypothesized to cause a generalized movement of the cytoplasmic domain toward the membrane, but the net effect is likely more complex, yielding a yet unpredictable motion of the slide-helix. Lipid tethering of the slide-helix to the membrane at residue $\mathrm{L} 51 \mathrm{C}$ activates the channel and would be expected to antagonize $\mathrm{PIP}_{2}$ inhibition if $\mathrm{PIP}_{2}$ also acted through a slide-helix displacement. The fact that lipid tethering does not alter $\mathrm{PIP}_{2}$ sensitivity suggests that $\mathrm{PIP}_{2}$ may have alternate transduction pathways outside of the slide-helix.

Covalent modification by hydrophobic moieties is a recognized mechanism for protein modulation in general, and palmitoylation may serve to tether various proteins, including ion channels (Hurley et al., 2000; Gubitosi-Klug et al., 2005; Resh, 2006), to lipid membranes, thereby influencing their location within the cell (Zhang and Casey, 1996; Boccuni et al., 2000; Peters et al., 2004; Smotrys and Linder, 2004). Direct changes of protein function as a result of such interactions have received less consideration. The present study demonstrates that for one particular class of ion channel, interaction of a specific domain, the slidehelix, with the membrane can directly control channel activity, and may illustrate a general principle by which cytoplasmic domain interactions with the membrane control gating of other channel types (Hilgemann and Ball, 1996; Baukrowitz et al., 1998; Shyng and Nichols, 1998; Runnels et al., 2002; Rohacs et al., 2005; Suh and Hille, 2005).

This work was supported by National Institutes of Health grant HL54171 (to C.G. Nichols) and DK69424 (to D. Enkvetchakul).

Lawrence G. Palmer served as editor. 
Submitted: 12 February 2007

Accepted: 23 July 2007

\section{REFERENCES}

Baukrowitz, T., U. Schulte, D. Oliver, S. Herlitze, T. Krauter, S.J. Tucker, J.P. Ruppersberg, and B. Fakler. 1998. PIP $_{2}$ and PIP as determinants for ATP inhibition of KATP channels. Science. 282:1141-1144.

Boccuni, P., L. Del Vecchio, R. Di Noto, and B. Rotoli. 2000. Glycosyl phosphatidylinositol (GPI)-anchored molecules and the pathogenesis of paroxysmal nocturnal hemoglobinuria. Crit. Rev. Oncol. Hematol. 33:25-43.

Enkvetchakul, D., and C.G. Nichols. 2003. Gating mechanism of KATP channels: function fits form. J. Gen. Physiol. 122:471-480.

Enkvetchakul, D., J. Bhattacharyya, I. Jeliazkova, D.K. Groesbeck, C.A. Cukras, and C.G. Nichols. 2004. Functional characterization of a prokaryotic Kir channel. J. Biol. Chem. 279:47076-47080.

Enkvetchakul, D., I. Jeliazkova, and C.G. Nichols. 2005. Direct modulation of Kir channel gating by membrane phosphatidylinositol 4,5-bisphosphate. J. Biol. Chem. 280:35785-35788.

Gloyn, A.L., E.R. Pearson, J.F. Antcliff, P. Proks, G.J. Bruining, A.S. Slingerland, N. Howard, S. Srinivasan, J.M. Silva, J. Molnes, et al. 2004. Activating mutations in the gene encoding the ATP-sensitive potassium-channel subunit Kir6.2 and permanent neonatal diabetes. N. Engl. J. Med. 350:1838-1849.

Gubitosi-Klug, R.A., D.J. Mancuso, and R.W. Gross. 2005. The human Kv1.1 channel is palmitoylated, modulating voltage sensing: identification of a palmitoylation consensus sequence. Proc. Natl. Acad. Sci. USA. 102:5964-5968.

Hilgemann, D.W., and R. Ball. 1996. Regulation of cardiac $\mathrm{Na}^{+}$, $\mathrm{Ca}^{2+}$ exchange and KATP potassium channels by $\mathrm{PIP}_{2}$. Science. 273:956-959.

Hurley, J.H., A.L. Cahill, K.P. Currie, and A.P. Fox. 2000. The role of dynamic palmitoylation in $\mathrm{Ca}^{2+}$ channel inactivation. Proc. Natl. Acad. Sci. USA. 97:9293-9298.

Khadikar, P.V., D. Mandloi, A.V. Bajaj, and S. Joshi. 2003. QSAR study on solubility of alkanes in water and their partition coefficients in different solvent systems using PI index. Bioorg. Med. Chem. Lett. 13:419-422.

Kuo, A., J.M. Gulbis, J.F. Antcliff, T. Rahman, E.D. Lowe, J. Zimmer, J. Cuthbertson, F.M. Ashcroft, T. Ezaki, and D.A. Doyle. 2003.
Crystal structure of the potassium channel KirBac1.1 in the closed state. Science. 300:1922-1926.

Peters, C., A. Wolf, M. Wagner, J. Kuhlmann, and H. Waldmann. 2004. The cholesterol membrane anchor of the Hedgehog protein confers stable membrane association to lipid-modified proteins. Proc. Natl. Acad. Sci. USA. 101:8531-8536.

Plaster, N.M., R. Tawil, M. Tristani-Firouzi, S. Canun, S. Bendahhou, A. Tsunoda, M.R. Donaldson, S.T. Iannaccone, E. Brunt, R. Barohn, et al. 2001. Mutations in Kir2.1 cause the developmental and episodic electrical phenotypes of Andersen's syndrome. Cell. 105:511-519.

Resh, M.D. 2006. Palmitoylation of ligands, receptors, and intracellular signaling molecules. Sci. STKE. 2006:re14.

Rohacs, T., C.M. Lopes, I. Michailidis, and D.E. Logothetis. 2005. PI $(4,5) \mathrm{P} 2$ regulates the activation and desensitization of TRPM8 channels through the TRP domain. Nat. Neurosci. 8:626-634.

Runnels, L.W., L. Yue, and D.E. Clapham. 2002. The TRPM7 channel is inactivated by PIP(2) hydrolysis. Nat. Cell Biol. 4:329-336.

Schulte, U., H. Hahn, M. Konrad, N. Jeck, C. Derst, K. Wild, S. Weidemann, J.P. Ruppersberg, B. Fakler, and J. Ludwig. 1999. pH gating of ROMK (K(ir) 1.1) channels: control by an Arg-Lys-Arg triad disrupted in antenatal Bartter syndrome. Proc. Natl. Acad. Sci. USA. 96:15298-15303.

Schulze, D., T. Krauter, H. Fritzenschaft, M. Soom, and T. Baukrowitz. 2003. Phosphatidylinositol 4,5-bisphosphate $\left(\mathrm{PIP}_{2}\right)$ modulation of ATP and $\mathrm{pH}$ sensitivity in Kir channels. A tale of an active and a silent $\mathrm{PIP}_{2}$ site in the $\mathrm{N}$ terminus. J. Biol. Chem. 278:10500-10505.

Shyng, S.L., and C.G. Nichols. 1998. Membrane phospholipid control of nucleotide sensitivity of KATP channels. Science. 282:1138-1141.

Smotrys, J.E., and M.E. Linder. 2004. Palmitoylation of intracellular signaling proteins: regulation and function. Annu. Rev. Biochem. 73:559-587.

Suh, B.C., and B. Hille. 2005. Regulation of ion channels by phosphatidylinositol 4,5-bisphosphate. Curr. Opin. Neurobiol. 15:370-378.

Sun, S., J.H. Gan, J.J. Paynter, and S.J. Tucker. 2006. Cloning and functional characterization of a superfamily of microbial inwardly rectifying potassium channels. Physiol. Genomics. 26:1-7.

Zhang, F.L., and P.J. Casey. 1996. Protein prenylation: molecular mechanisms and functional consequences. Annu. Rev. Biochem. 65:241-269. 